\title{
A new hyperjerk dynamical system with hyperchaotic attractor and two saddle-focus rest points exhibiting Hopf bifurcations, its hyperchaos synchronisation and circuit implementation
}

\section{Sundarapandian Vaidyanathan*}

Research and Development Centre

Vel Tech University, Chennai-600062, Tamil Nadu, India

E-mail: sundarcontrol@gmail.com

*Corresponding author

\section{Irene M. Moroz}

Mathematical Institute

University of Oxford

Andrew Wiles Building, ROQ

Oxford Ox2 6GG, UK

E-mail: Irene.Moroz@maths.ox.ac.uk

\section{Aceng Sambas}

Department of Mechanical Engineering

Universitas Muhammadiyah Taskimalaya

Tasikmalaya, Jawa Barat 46196, Indonesia

E-mail: acengs@umtas.ac.id

\begin{abstract}
A new 4-D dynamical hyperjerk system with hyperchaos is reported in this work. The proposed nonlinear mechanical system with hyperchaos has two saddlefocus rest points exhibiting Hopf bifurcations. A detailed bifurcation analysis of the new hyperjerk plant with theory and simulations is discussed. As a control application, an integral sliding mode controller has been designed for the global hyperchaos synchronisation of the new hyperjerk system with itself. Finally, a circuit model using MultiSim of the new hyperjerk system with hyperchaos is designed for practical implementation.
\end{abstract}

Keywords: Hyperchaos; hyperjerk; sliding mode control; synchronisation; circuit design.

Reference to this paper should be made as follows: Vaidyanathan, S., Moroz, I.M., and Sambas, A. (2019) 'A new hyperjerk dynamical system with hyperchaotic attractor and two saddle-focus rest points exhibiting Hopf bifurcation, its hyperchaos synchronisation and circuit implementation ', Int. J. Modelling, Identification and Control, Vol. x, No. x, pp. $x x x-x x x$.

\section{Biographical notes:}

Sundarapandian Vaidyanathan is a Professor and Dean at the R \& D Centre, Vel Tech University, Chennai, India. He received his D.Sc in Electrical and Systems Engineering from Washington University, St. Louis, USA in 1996. He has published over 490 Scopus-indexed research papers. His current research focuses on control systems, chaos theory, chaotic and hyperchaotic systems, sliding mode control, neuro-fuzzy control, computational science, circuits and memristors. He is the Editor-in-Chief of International Journal of Nonlinear Dynamics and Control (IJNDC), Inderscience Publishers, Olney, UK. He is also in the Editorial Boards of many control journals published by Inderscience, Olney, UK.

Irene Moroz graduated with a Class I in Mathematics from Oxford University in 1977. She then received her $\mathrm{PhD}$ on slowly varying baroclinic waves from Leeds University, UK in 1981. After spending 2 years as a post-doctoral fellow in Leeds, she spent 18 months as a Visiting Assistant Professor at Cornell University, before taking up a New Blood lectureship at the University of East Anglia in 1985. Since 1992, she has been a Fellow in Applied Mathematics at St Hilda's College, Oxford. Her research interests include Dynamical Systems, Mathematical Ecology, Geophysical Fluid Dynamics, Dynamo models and climate modelling.

Aceng Sambas is currently a Lecturer at the Muhammadiyah University of Tasikmalaya, Indonesia since 2015. He received his MSc in Mathematics from the Universiti Sultan Zainal Abidin (UniSZA), Malaysia in 2015. His current research focuses on dynamical systems, chaotic signals, electrical engineering, computational science, signal processing, robotics, embedded systems and artificial intelligence. 


\section{Introduction}

Some miscellaneous topics of research in the control literature are the modelling, identification and control of systems (Leandro and Kienitz, 2019; Tirandaz, 2019; Habrache et al., 2019; Fadil et al., 2019; Wang, 2019; Abdelhedi and Derbel, 2019; Lien et al., 2019a,b; Vaidyanathan et al., 2019b). Significant research attention has been devoted to the modelling and applications of dynamical systems exhibiting chaos and hyperchaos (Xu et al., 2019; Gusso et al., 2019; Gatabazi et al., 2019; Singh and Roy, 2019; Cabanas et al., 2019; Ginoux et al., 2019; Jahanshahi et al., 2019; Rajagopal et al., 2019b; Daumann and Rech, 2019). $\mathrm{Xu}$ et al. (2019) proposed a chaotic system based on a circuit design involving a memristor model and a meminductor model. Gusso et al. (2019) analyzed the nonlinear dynamical model and the existence of chaos in suspended beam micro/nanoelectromechanical (MEMS/NEMS) resonators that are actuated by twosided electrodes. Gatabazi et al. (2019) analyzed 2-D and 3-D Grey Lotka-Volterra Models (GLVM) and explored their application in cryptocurrencies such as Bitcoin, Litecoin and Ripple. Singh and Roy (2019) studied microscopic chaos control of a chemical reactor system via nonlinear active plus proportional integral sliding mode control. Cabanas et al. (2019) discovered chaos in driven nano-magnets such as spin valves by using the magnetic energy and the magnetoresistance. Ginoux et al. (2019) discovered chaos in a dynamical system modelling the illicit drug consumption in a population comprising drug users and non-users. Jahanshahi et al. (2019) discussed a finance hyperchaos system via entropy analysis and control methods. Rajagopal et al. (2019) proposed a new van der Pol-Duffing (MVPD) snap oscillator exhibiting hyperchaos and implemented the hyperchaos system in FPGA. Daumann and Rech (2019) reported hyperchaos in a heat-flux convection model.

Jerk and hyperjerk dynamical systems are important classes of mechanical systems. If $y(t)$ denotes the displacement of a moving object, then $D y(t)=\frac{d y}{d t}$ represents its velocity, $D^{2} y(t)=\frac{d^{2} y}{d t^{2}}$ its acceleration, $D^{3} y(t)=\frac{d^{3} y}{d t^{3}}$ its jerk and $D^{4} y(t)=\frac{d^{4} y}{d t^{4}}$ its hyperjerk.

An autonomous jerk differential equation has the general representation given by

$$
D^{3} y=F\left(y, D y, D^{2} y\right)
$$

An autonomous hyperjerk differential equation has the general representation given by

$$
D^{4} y=F\left(y, D y, D^{2} y, D^{3} y\right)
$$

The hyperjerk differential equation (2) can be displayed in a system form as

$$
\left\{\begin{array}{l}
\dot{y}_{1}=y_{2} \\
\dot{y}_{2}=y_{3} \\
\dot{y}_{3}=y_{4} \\
\dot{y}_{4}=F\left(y_{1}, y_{2}, y_{3}, y_{4}\right)
\end{array}\right.
$$

Copyright (c) 201X Inderscience Enterprises Ltd.
Recently, good interest has been devoted to the finding of both jerk and hyperjerk systems in the chaos literature (Vaidyanathan et al., 2018a; Vaidyanathan, 2017; Vaidyanathan et al., 2017; Vaidyanathan, 2016, 2015e; El-Nabulsi, 2018; Prousalis et al., 2018; Ahmad and Srisuchinwong, 2018; Tsafack and Kengne, 2018; Daltzis et al., 2018; Vaidyanathan et al., 2018b; Wang et al., 2017). Vaidyanathan et al. (2018a) reported a new chaotic jerk system with two quadratic nonlinearities and discussed its applications to electronic circuit implementation and image encryption. Vaidyanathan (2017) reported a new 3 -D chaotic jerk system with two cubic nonlinear terms and discussed its adaptive synchronisation using backstepping control. Vaidyanathan et al. (2017) analyzed a new chaotic jerk system with its applications for circuit simulation and voice encryption. Vaidyanathan (2016) announced a new chaotic jerk system and discussed its adaptive synchronisation using backstepping control. Vaidyanathan (2015) proposed a new chaotic jerk system with two quadratic nonlinearities.

El-Nabulsi (2018) reported jerk and hyperjerk systems in the study of nonlocal effects in fluids, plasmas and solar physics. Prousalis et al. (2018) observed extreme multi-stability in a 4-D hyperjerk memristive system with infinite number of rest points. Ahmad and Srisuchinwong (2018) reported a 4-D hyperjerk system with hyperchaos and having no rest point. Tsafack and Kengne (2018) reported a 5-D hyperjerk system with circuit design. Daltzis et al. (2018) reported a 4D hyperjerk system with hyperchaos and built a real circuit design for the hyperjerk system. Vaidyanathan et al. (2018b) reported a new 4-D chaotic hyperjerk system and discussed its applications in RNG, image encryption and steganography. Wang et al. (2017) found a new 4-D hyperchaotic hyperjerk system with coexisting attractors.

This work reports a new 4-D dynamical hyperjerk system with hyperchaos. The proposed nonlinear mechanical system with hyperchaos has two nonlinear terms. A novel feature of the proposed hyperjerk system is the existence of simple Hopf bifurcations emerging from the two saddle-focus equilibrium points of the system. A detailed bifurcation analysis of the new hyperjerk plant with theory and simulations is discussed.

The synchronisation problem of the chaos theory is to consider a pair of chaotic systems designated as the master and slave systems and the design goal is to build feedback control using their state trajectories so as to drive the states of the slave system to asymptotically track those of the master system asymptotically (Vaidyanathan, 2015g; Sundarapandian, 2013). Many control strategies are available to deal with the control and synchronisation of chaotic systems such as the active control (Vaidyanathan, 2015f, 2013, 2011a,b), adaptive control (Vaidyanathan, 2012, 2015c,d,i,h,a), passive control (Vaidyanathan et al., 2019c; Gritli and Belghith, 2016), backstepping control (Vaidyanathan 
et al., 2015; Rasappan and Vaidyanathan, 2012; Ricci et al., 2018), fuzzy control (Vaidyanathan and Azar, 2016; Boulkroune et al., 2016), etc.

Utkin and Shi (1996) proposed an improved sliding control method named integral sliding mode control (ISMC). In contrast with conventional sliding mode control, the system motion under integral sliding mode has a dimension equal to that of the state space. In ISMC, the system trajectory always starts from the sliding surface. Accordingly, the reaching phase is eliminated, and robustness in the whole state space is assured (Utkin and Shi, 1996).

In this work, we use Integral Sliding Manifold Control (ISMC) for the global hyperchaos synchronisation of the new hyperjerk system with itself. Sliding mode controllers have many advantages such as robustness, fast convergence, etc., and have many applications in science and engineering (Vaidyanathan, 2015b; Azar and Zhu, 2015; Vaidyanathan and Volos, 2016; Vaidyanathan and Lien, 2017).

Finally, a circuit model using MultiSim of the new hyperjerk system with hyperchaos is designed for practical implementation. We show that the MultiSim outputs of the hyperjerk system exhibit a good match with the MATLAB simulations of the same system. Circuit realizations of chaos and hyperchaos dynamical systems are useful for real-world implementations (Rajagopal et al., 2019a; Nwachioma et al., 2019; Vaidyanathan et al., 2019a).

\section{A new hyperjerk model}

In this work, we propose a new hyperjerk model with the 4-D dynamics

$$
\left\{\begin{array}{l}
\dot{x}=y \\
\dot{y}=z \\
\dot{z}=w \\
\dot{w}=-x-y-a x^{2}-b z+c w-x^{2}|x| w
\end{array}\right.
$$

which consists of two nonlinear terms in the fourth differential equation.

To simplify the notation, we denote the state vector as $X=(x, y, z, w)$ and the parameter set as $A=(a, b, c)$.

We shall show that the hyperjerk model (4) exhibits hyperchaos when $A$ takes the values

$$
A=(a, b, c)=(0.3,3.6,0.1)
$$

The Lyapunov exponents of the 4-D hyperjerk model (4) for the initial conditions $X(0)=(0.1,0,0.1,0)$ and the parameter values $(a, b, c)=(0.3,3.6,0.1)$ can be computed as

$$
\left\{\begin{array}{l}
L E_{1}=0.1158 \\
L E_{2}=0.0251 \\
L E_{3}=0 \\
L E_{4}=-1.1086
\end{array}\right.
$$

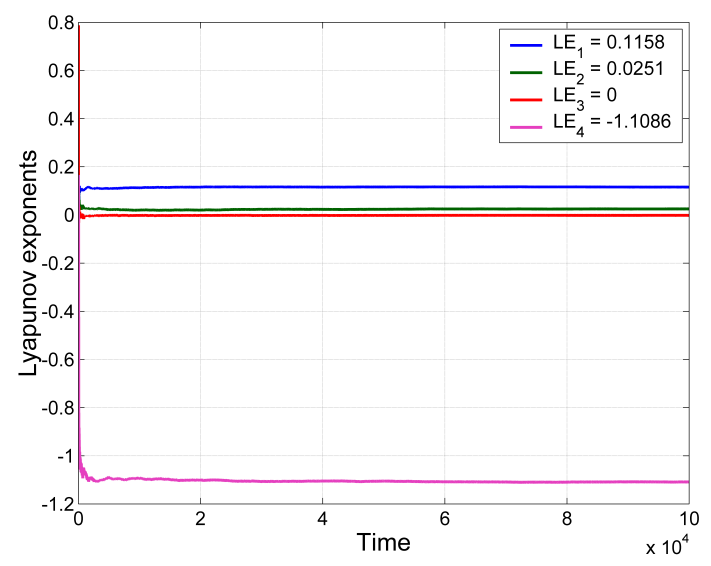

Figure 1: MATLAB simulation showing the Lyapunov exponents of the new hyperjerk model (4)

Figure 1 shows the estimation of the Lyapunov exponents of the hyperjerk model (4).

Since $L E_{1}$ and $L E_{2}$ are positive and $L E_{1}+L E_{2}+$ $L E_{3}+L E_{4}<0$, it is deduced that the 4 -D hyperjerk model (4) has a dissipative and hyperchaotic attractor. Furthermore, $D_{K Y}$, the Kaplan-Yorke dimension of the hyperjerk model (4) can be estimated as

$$
D_{K Y}=3+\frac{L E_{1}+L E_{2}+L E_{3}}{\left|L E_{4}\right|}=3.1271
$$

Figures 2-5 give MATLAB simulations of the 4D hyperjerk model (4) for the hyperchaos case when $(a, b, c)=(0.3,3.6,0.1)$ and the initial state $X(0)=$ $(0.1,0,0.1,0)$.

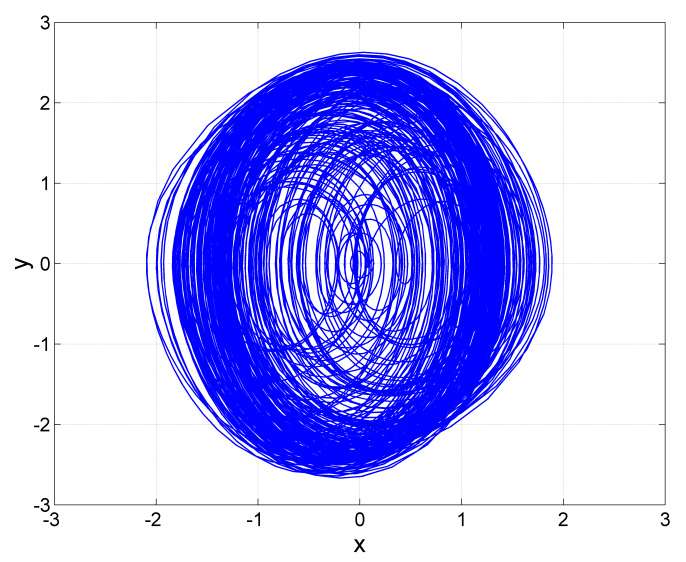

Figure 2: MATLAB simulation showing the $(x, y)$ planar plot of the hyperjerk model (4) depicting hyperchaos for $X(0)=(0.1,0,0.1,0)$ and parameter values $(a, b, c)=(0.3,3.6,0.1)$.

\section{Dynamic analysis of the hyperjerk system}

The four-dimensional hyperjerk system is

$$
\dot{x}=y
$$




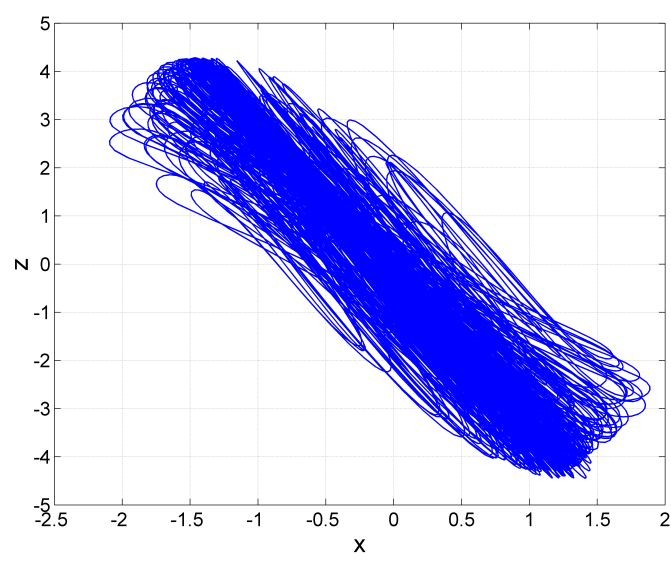

Figure 3: MATLAB simulation showing the $(x, z)$ planar plot of the hyperjerk model (4) depicting hyperchaos for $X(0)=(0.1,0,0.1,0)$ and parameter values $(a, b, c)=(0.3,3.6,0.1)$.

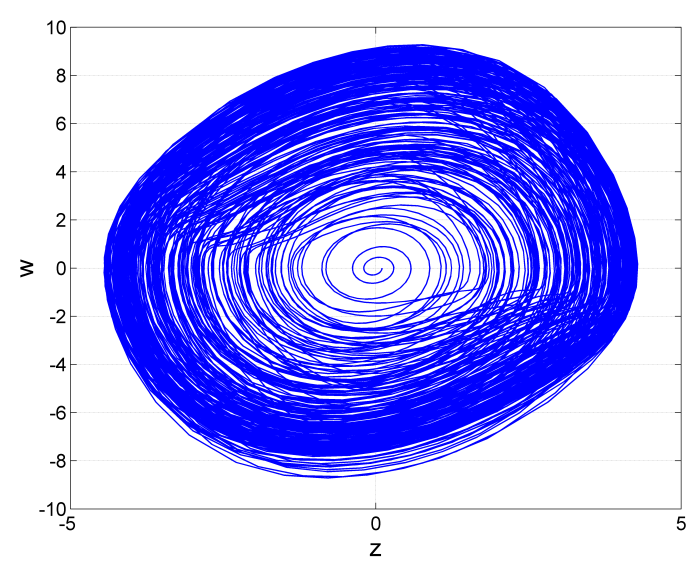

Figure 4: MATLAB simulation showing the $(z, w)$ planar plot of the hyperjerk model (4) depicting hyperchaos for $X(0)=(0.1,0,0.1,0)$ and parameter values $(a, b, c)=(0.3,3.6,0.1)$.

$$
\begin{aligned}
\dot{y} & =z \\
\dot{z} & =w \\
\dot{w} & =-x-y-a x^{2}-b z+c w-x^{2}|x| w
\end{aligned}
$$

While the chosen set of parameter values are $(a, b, c)=$ $(0.3,3.6,0.1)$, we find it instructive to explore the dynamics of system (8) for other parameter values. In this context, we find the equilibrium states and determine which bifurcations are possible.

The equilibrium states are found by setting the righthand-side (RHS) of (8) to zero. Thus, we get

$$
\begin{aligned}
y & =0 \\
z & =0 \\
w & =0 \\
-x-y-a x^{2}-b z+c w-x^{2}|x| w & =0
\end{aligned}
$$

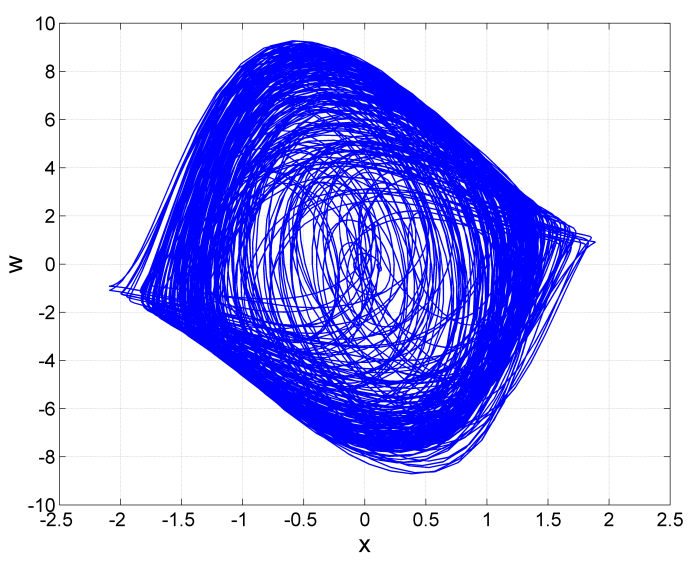

Figure 5: MATLAB simulation showing the $(x, w)-$ planar plot of the hyperjerk model (4) depicting hyperchaos for $X(0)=(0.1,0,0.1,0)$ and parameter values $(a, b, c)=(0.3,3.6,0.1)$.

From (9a), (9b) and (9c), we see that

$$
y=0, z=0, w=0
$$

Substituting these values into $(9 \mathrm{~d})$, we get

$$
-x-a x^{2}=0 \text { or }-x(1+a x)=0
$$

Hence, we obtain two equilibria: the trivial fixed point $(x, y, z, w)_{0}=(0,0,0,0)$ and a nontrivial equilibrium state: $(x, y, z, w)_{e}=(0,0,0,-1 / a)$.

The linear stability of the equilibrium states is found by computing the fourth order Jacobian matrix:

$$
J=\left(\begin{array}{cccc}
0 & 1 & 0 & 0 \\
0 & 0 & 1 & 0 \\
0 & 0 & 0 & 1 \\
-1-2 a x-3 s w x^{2} & -1 & -b c-s x^{3}
\end{array}\right),
$$

where $s=1$ if $x \geq 0$ and $s=-1$ if $x<0$. Linear stability is found by computing the determinant of $J-\lambda I_{4}$ for each equilibrium state where $\lambda$ give the eigenvalues and $I_{4}$ is the $(4,4)$ identity matrix.

(i) If $x=0$, we obtain the quartic characteristic equation:

$$
\lambda^{4}-c \lambda^{3}+b \lambda^{2}+\lambda+1=0 .
$$

(ii) If $x=-1 / a$, we obtain the quartic characteristic equation:

$$
\lambda^{4}-\left(c+s / a^{3}\right) \lambda^{3}+b \lambda^{2}+\lambda-1=0 .
$$

\subsection{Bifurcations}

No steady bifurcations are possible, since $\lambda=0$ cannot be a root of either (13) or (14). Thus, we consider the possibility of simple Hopf bifurcations. (Multiple Hopf bifurcations are also not possible, because the coefficient of $\lambda$ in these equations cannot vanish.) 
We firstly investigate whether the trivial equilibrium solution undergoes a Hopf bifurcation by substituting $\lambda=i \omega$ into (13). This gives the pair of equations

$$
\omega^{4}-b \omega^{2}+1=0, \quad c \omega^{2}+1=0 .
$$

The second of these equations gives $\omega^{2}=-1 / c>0$, so that $c$ must be negative, and the frquency of the oscillation at bifurcation is given by $\omega=\sqrt{-1 / c}$. For real roots to the first equation we require

$$
\omega^{2}=\frac{b}{2} \pm \frac{1}{2}\left[b^{2}-4\right]^{1 / 2},
$$

so that $b>2$ for real solutions. Substituting $\omega^{2}=-1 / c$ into the first equation in (15) gives

$$
c^{2}+b c+1=0
$$

which gives two possible values for $c$ :

$$
c=\frac{1}{2}\left(-b \pm \sqrt{\left[b^{2}-4\right]}\right) .
$$

When $b=3.6,(17)$ gives $c_{H B+}=-0.3033$ and $c_{H B-}=$ -3.2966 , with frequencies from (15) of $\omega_{+}=0.5507$ and $\omega_{-}=1.8157$ respectively. Figure 6 shows the variation of the complex eigenvalues as $c$ varies. The two Hopf bifurcations, labelled as $H B_{ \pm}$are given by the two roots of $(16)$.

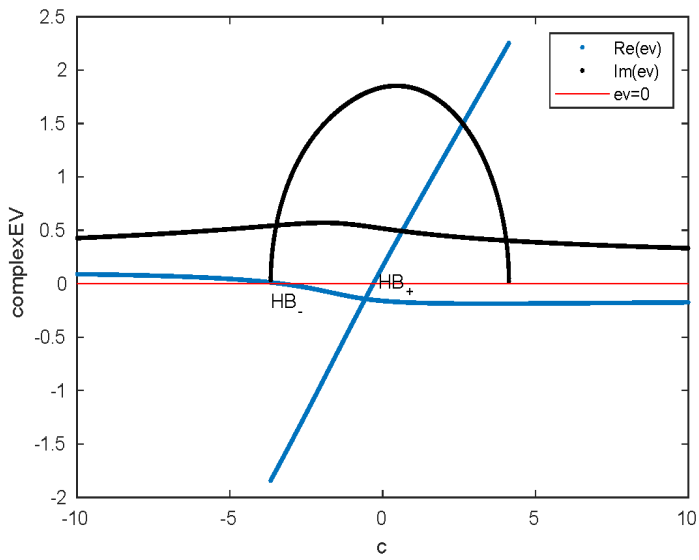

Figure 6: A bifurcation plot of the complex eigenspectrum of (13) as $c$ varies. There are two Hopf bifurcation points at which the real part of the complex eigenvalue crosses the imaginary axis (shown as the blue curve crossing the red line). When the imaginary part of the spectrum terminates on the zero line, all the eigenvalues become real.

A Hopf bifurcation is also possible for the nontrivial equilibrium state $x=-1 / a$. Substituting $\lambda=i \Omega$ into (14), and again separating into real and imaginary parts, we obtain

$$
\Omega^{4}-b \Omega^{2}-1=0, \quad\left(c+s / a^{3}\right) \Omega^{2}+1=0,
$$

which combine to give

$$
c+s / a^{3}=\frac{1}{2}\left(b \pm \sqrt{\left[b^{2}+4\right]}\right) .
$$

We can either fix $b$ and $a$ to determine $c$ or fix $b$ and $c$ to determine $a$ for the existence of a Hopf Bifurcation. Since $\Omega^{2}$ must be positive in the second equation of (18), we must take $c+s / a^{3}<0$. This implies that $s=-1$, so that $x<0$ and $a>0$. From (19), we must therefore take the negative square root, since $\sqrt{\left[b^{2}+4\right]}>b$, and there is only one choice for the Hopf Bifurcation. If we fix $a=0.3$ and $c=0.1$, we find that $b_{H}=-36.91$. If we fix $a=0.3$ and $b=3.6$, we find that $c_{H B}=36.778$, whereas if we fix $b=3.6$ and $c=0.1$, we obtain $a=$ 1.407, with a corresponding frequency of $\Omega=1.9645$. Figure 7 shows the corresponding spectrum for the nontrivial equilibrium state, as $a$ varies for $b=3.6$ and $c=0.1$. Because of the modulus sign in $(9 \mathrm{~d})$, the curves are reflectionally symmetric about $a=0$.

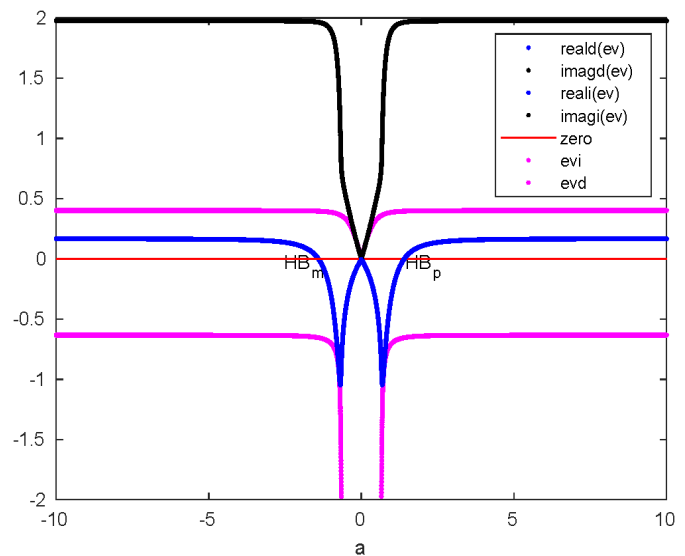

Figure 7: This figure is similar to Figure 6, but it is plotted as a varies, and includes the real eigenvalues (magenta) as well as the complex eigenspectrum.

Figure 8 shows the variation of real and complex eigenvalues for the nontrivial equilibrium state as $c$ varies for $a=0.3$ and $b=3.6$. Note the range of $c$ values lies well beyond the chosen parameter value for $c$ of $c=0.1$.

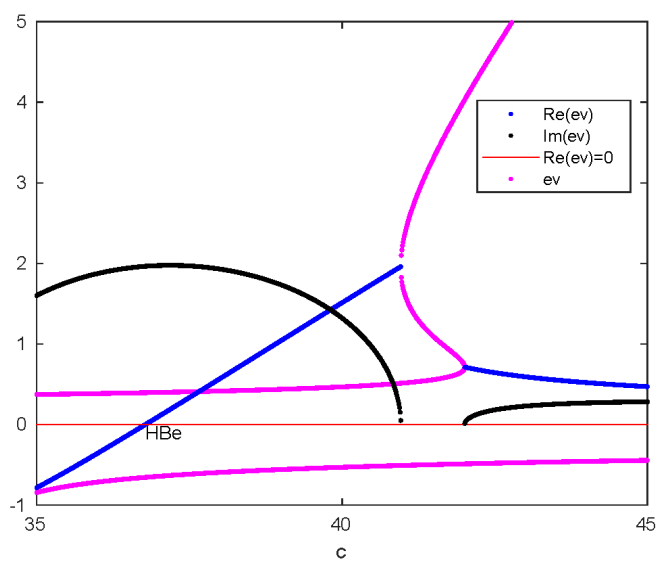

Figure 8: This figure is similar to Figure 6, but it is plotted as $c$ varies, and includes the real eigenvalues (magenta) as well as the complex eigenspectrum. 


\subsection{Numerical integrations}

Figure 9 shows a bifurcation transition plot of $x_{\max }$ as $c$ varies. There are two regimes. A chaotic region for $c>0$ undergoes a period halving bifurcation to create a periodic solution, which disappears in the Hopf bifurcation when $c=-0.304$. For $-3.297<c<-0.304$, we only find stable steady states. The second Hopf bifurcation at $c=-3.297$ gives rise to period-doubling and halving bifurcations. When we tried to continue the integrations beyond the two end points of the plot, we found that the system became unbounded as all three variables grew.

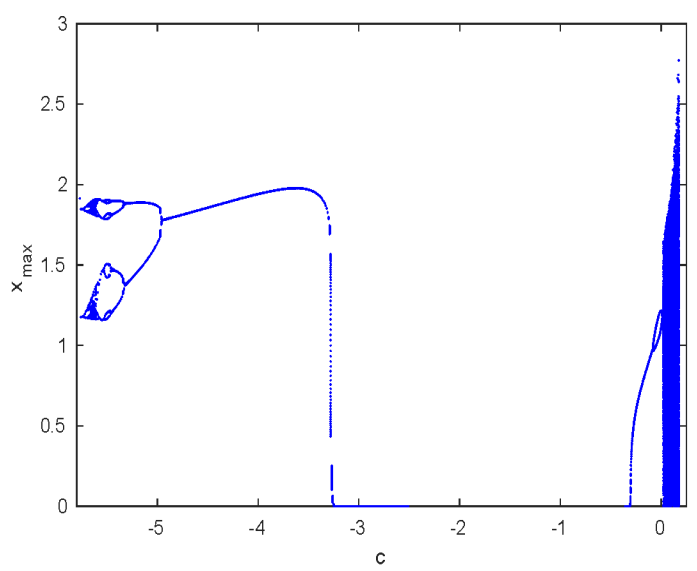

Figure 9: Part of the bifurcation transition plot of $x_{\max }$ as $c$ varies. Clearly shown are the two Hopf bifurcation values, derived in the previous section.

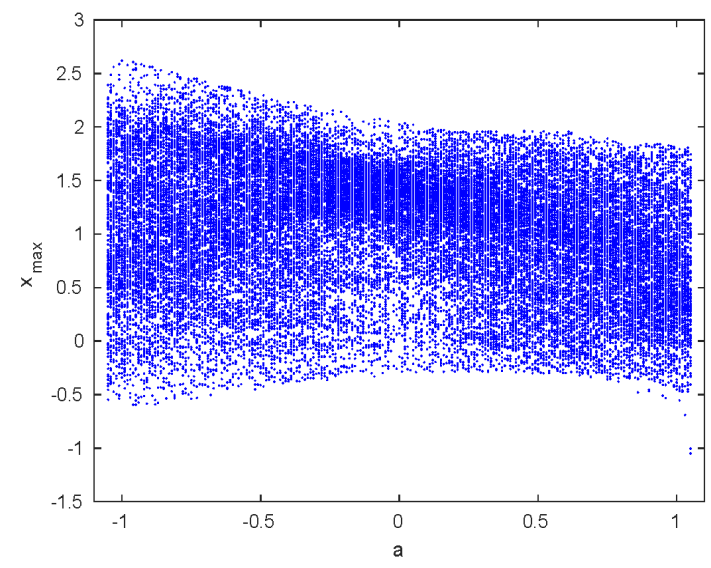

Figure 10: Bifurcation transition plot of $x_{\max }$ as $a$ varies.

Figure 10 shows the bifurcation transition plot of the maxima of $x$ over each cycle as a varies. From the pervious section, we saw that the nontrivial fixed point can undergo a Hopf bifurcation when $a=1.407$ for $b=3.6$ and $c=0.1$. The range of chaotic states in Figure 4 lies well inside $-1.407<a<1.407$. If we set $a=1.4$, system (1.1) evolves to a state in which both $x$ and $y$ grow without bound, $z \rightarrow-1.76718$ and $w \rightarrow 0$. Instead if we set $a=1.41$, both $x$ and $y$ again evolve without bound, $z \rightarrow-1.7743$ and again $w \rightarrow 0$. These values fall on either side of the Hopf bifurcation value for the nontrivial fixed point. We conclude that, in contrast to the trivial fixed point, this Hopf bifurcation is subcritical.

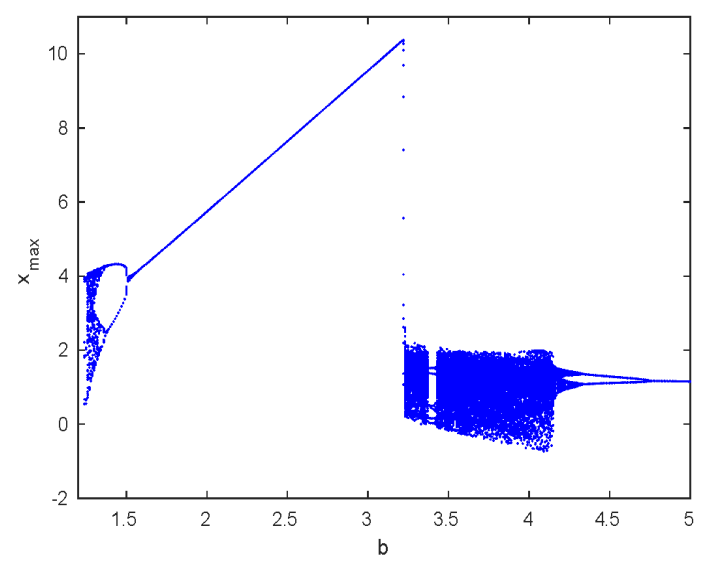

Figure 11: Bifurcation transition plot of $x_{\max }$ as $b$ varies.

Figure 11 shows the bifurcation transition plot of $x_{\max }$ as $b$ varies. For the chosen set of parameter values, we see that the dynamics falls within the chaotic region. There are other regions for $b$ that give rise to periodic states $(1.5<b<3.2)$ or mutiply periodic solutions, such as period-two cycles. Numerical integrations show that the periodic solution continues beyond $b=15$.

\section{Hyperchaos synchronisation of the new hyperjerk dynamical model}

As a control application, this section details the control design of achieving global hyperchaos synchronisation of the new 4-D hyperjerk dynamical model (4) with itself (drive-response systems) via integral sliding mode control (ISMC).

As the drive system, we focus on the nonlinear plant

$$
\left\{\begin{array}{l}
\dot{x}_{1}=y_{1} \\
\dot{y}_{1}=z_{1} \\
\dot{z}_{1}=w_{1} \\
\dot{w}_{1}=-x_{1}-y_{1}-a x_{1}^{2}-b z_{1}+c w_{1}-x_{1}^{2}\left|x_{1}\right| w_{1}
\end{array}\right.
$$

where $X_{1}=\left(x_{1}, y_{1}, z_{1}, w_{1}\right)$ is the state and $a, b$ are constant parameters. 
As the slave system of the synchronisation process, we focus on the nonlinear plant

$$
\left\{\begin{aligned}
\dot{x}_{2}= & y_{2}+\nu_{x} \\
\dot{y}_{2}= & z_{2}+\nu_{y} \\
\dot{z}_{2}= & w_{2}+\nu_{z} \\
\dot{w}_{2}= & -x_{2}-y_{2}-a x_{2}^{2}-b z_{2}+c w_{2} \\
& \quad-x_{2}^{2}\left|x_{2}\right| w_{2}+\nu_{w}
\end{aligned}\right.
$$

where $X_{2}=\left(x_{2}, y_{2}, z_{2}, w_{2}\right)$ is the state and $\nu_{x}, \nu_{y}, \nu_{z}, \nu_{w}$ serve as the sliding controls.

Next, we define the synchronisation error between the drive system (20) and the response system (21) in the following manner:

$$
\left\{\begin{array}{l}
\epsilon_{x}=x_{2}-x_{1} \\
\epsilon_{y}=y_{2}-y_{1} \\
\epsilon_{z}=z_{2}-z_{1} \\
\epsilon_{w}=w_{2}-w_{1}
\end{array}\right.
$$

The synchronisation error dynamics can be easily determined as follows:

$$
\left\{\begin{aligned}
\dot{\epsilon}_{x}= & \epsilon_{y}+\nu_{x} \\
\dot{\epsilon}_{y}= & \epsilon_{z}+\nu_{y} \\
\dot{\epsilon}_{z}= & \epsilon_{w}+\nu_{z} \\
\dot{\epsilon}_{w}= & -\epsilon_{x}-\epsilon_{y}-b \epsilon_{z}+c \epsilon_{w}-a\left(x_{2}^{2}-x_{1}^{2}\right) \\
& -x_{2}^{2}\left|x_{2}\right| w_{2}+x_{1}^{2}\left|x_{1}\right| w_{1}+\nu_{w}
\end{aligned}\right.
$$

The integral sliding surface connected with each error state can be classified according to the following equations.

$$
\left\{\begin{array}{l}
\sigma_{x}=\epsilon_{x}+\lambda_{x} \int_{0}^{t} \epsilon_{x}(\tau) d \tau \\
\sigma_{y}=\epsilon_{y}+\lambda_{y} \int_{0}^{t} \epsilon_{y}(\tau) d \tau \\
\sigma_{z}=\epsilon_{z}+\lambda_{z} \int_{0}^{t} \epsilon_{z}(\tau) d \tau \\
\sigma_{w}=\epsilon_{w}+\lambda_{w} \int_{0}^{t} \epsilon_{w}(\tau) d \tau
\end{array}\right.
$$

Using (24), we derive the differential equations of the integral sliding surfaces as follows:

$$
\left\{\begin{array}{l}
\dot{\sigma}_{x}=\dot{\epsilon}_{x}+\lambda_{x} \epsilon_{x} \\
\dot{\sigma}_{y}=\dot{\epsilon}_{y}+\lambda_{y} \epsilon_{y} \\
\dot{\sigma}_{z}=\dot{\epsilon}_{z}+\lambda_{z} \epsilon_{z} \\
\dot{\sigma}_{w}=\dot{\epsilon}_{w}+\lambda_{w} \epsilon_{w}
\end{array}\right.
$$

We suppose that $\lambda_{x}, \lambda_{y}, \lambda_{z}, \lambda_{w}$ are positive constants. Thus, the Hurwitz condition is satisfied.
Using integral sliding mode control (Vaidyanathan and Lien, 2017), we take the feedback control as

$$
\left\{\begin{aligned}
\nu_{x}= & -\epsilon_{y}-\lambda_{x} \epsilon_{x}-\tau_{x} \operatorname{sgn}\left(\sigma_{x}\right)-\kappa_{x} \sigma_{x} \\
\nu_{y}= & -\epsilon_{z}-\lambda_{y} \epsilon_{y}-\tau_{y} \operatorname{sgn}\left(\sigma_{y}\right)-\kappa_{y} \sigma_{y} \\
\nu_{z}= & -\epsilon_{w}-\lambda_{z} \epsilon_{z}-\tau_{z} \operatorname{sgn}\left(\sigma_{z}\right)-\kappa_{z} \sigma_{z} \\
\nu_{w}= & \epsilon_{x}+\epsilon_{y}+b \epsilon_{z}-c \epsilon_{w}+a\left(x_{2}^{2}-x_{1}^{2}\right) \\
& +x_{2}^{2}\left|x_{2}\right| w_{2}-x_{1}^{2}\left|x_{1}\right| w_{1} \\
& -\lambda_{w} \epsilon_{w}-\tau_{w} \operatorname{sgn}\left(\sigma_{w}\right)-\kappa_{w} \sigma_{w}
\end{aligned}\right.
$$

Substitution of the control law (26) into (23) results in the closed-loop error system

$$
\left\{\begin{array}{l}
\dot{\epsilon}_{x}=-\lambda_{x} \epsilon_{x}-\tau_{x} \operatorname{sgn}\left(\sigma_{x}\right)-\kappa_{x} \sigma_{x} \\
\dot{\epsilon}_{y}=-\lambda_{y} \epsilon_{y}-\tau_{y} \operatorname{sgn}\left(\sigma_{y}\right)-\kappa_{y} \sigma_{y} \\
\dot{\epsilon}_{z}=-\lambda_{z} \epsilon_{z}-\tau_{z} \operatorname{sgn}\left(\sigma_{z}\right)-\kappa_{z} \sigma_{z} \\
\dot{\epsilon}_{w}=-\lambda_{w} \epsilon_{w}-\tau_{w} \operatorname{sgn}\left(\sigma_{w}\right)-\kappa_{w} \sigma_{w}
\end{array}\right.
$$

We apply Lyapunov stability theory (Vaidyanathan and Lien, 2017) to establish the main control result of this section, which is stated in the following theorem.

Theorem 1: The new 4-D hyperjerk systems exhibiting hyperchaos represented by (20) and (21) are globally and completely synchronised for all initial states by the integral SMC law (26) where $\lambda_{x}, \lambda_{y}, \lambda_{z}, \lambda_{w}, \kappa_{x}$, $\kappa_{y}, \kappa_{z}, \kappa_{w}, \tau_{x}, \tau_{y}, \tau_{z}, \tau_{w}$ are taken as positive constants.

Proof. As the Lyapunov function, we consider

$$
V\left(\sigma_{x}, \sigma_{y}, \sigma_{z}, \sigma_{w}\right)=\frac{1}{2}\left(\sigma_{x}^{2}+\sigma_{y}^{2}+\sigma_{z}^{2}+\sigma_{w}^{2}\right)
$$

It is quite evident to observe that $V$ is a positive definite, quadratic, function on $\mathbf{R}^{4}$.

The time-derivative of the function $V$ along the error trajectories is calculated using (27) and (25) as follows:

$$
\begin{aligned}
\dot{V}= & -\tau_{x}\left|\sigma_{x}\right|-\tau_{y}\left|\sigma_{y}\right|-\tau_{z}\left|\sigma_{z}\right|-\tau_{w}\left|\sigma_{w}\right| \\
& -\kappa_{x} \sigma_{x}^{2}-\kappa_{y} \sigma_{y}^{2}-\kappa_{z} \sigma_{z}^{2}-\kappa_{w} \sigma_{w}^{2}
\end{aligned}
$$

Thus, $\dot{V}$ is a negative definite function defined on $\mathbf{R}^{4}$.

Hence, the proof is complete by Lyapunov stability theory (Vaidyanathan and Lien, 2017).

For numerical simulations in MATLAB, we consider the parameters as in the hyperchaos case for the hyperjerk master-slave systems, viz. $(a, b, c)=$ $(0.3,3.6,0.1)$.

The sliding constants are chosen so as to meet the sliding condition as well as to ensure fast convergence of the synchronization errors. Thus, we take $\kappa_{x}=\kappa_{y}=$ $\kappa_{z}=\kappa_{w}=10$.

We also take $\tau_{x}=\tau_{y}=\tau_{z}=\tau_{w}=0.1$.

Furthermore, we take $\lambda_{x}=\lambda_{y}=\lambda_{z}=\lambda_{w}=20$.

The initial condition of the master system (20) is taken as $x_{1}(0)=5.2, y_{1}(0)=3.9, z_{1}(0)=7.5$ and $w_{1}(0)=4.1$.

The initial condition of the slave system (21) is taken as $x_{2}(0)=2.7, y_{2}(0)=8.4, z_{2}(0)=3.4$ and $w_{2}(0)=1.9$. 
Figures 12-16 illustrate the sliding controller based global synchronisation between the two hyperjerk systems (20) and (21).

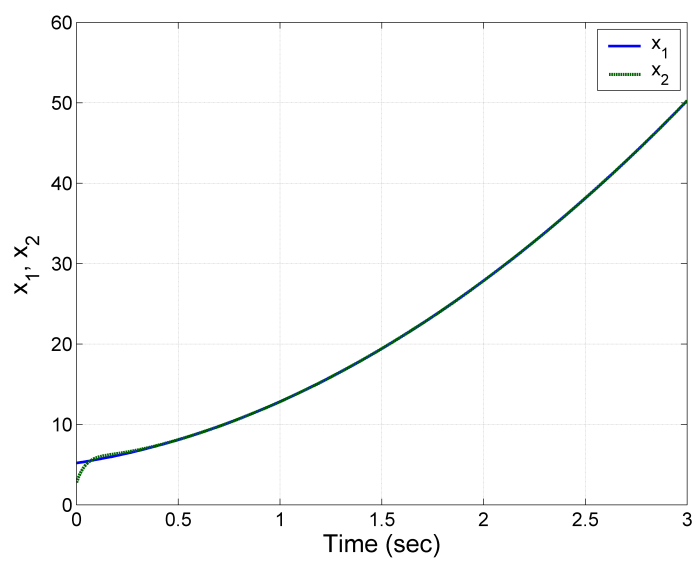

Figure 12: MATLAB simulation showing the synchronisation of the states $x_{1}$ and $x_{2}$ of the hyperjerk systems (20) and (21).

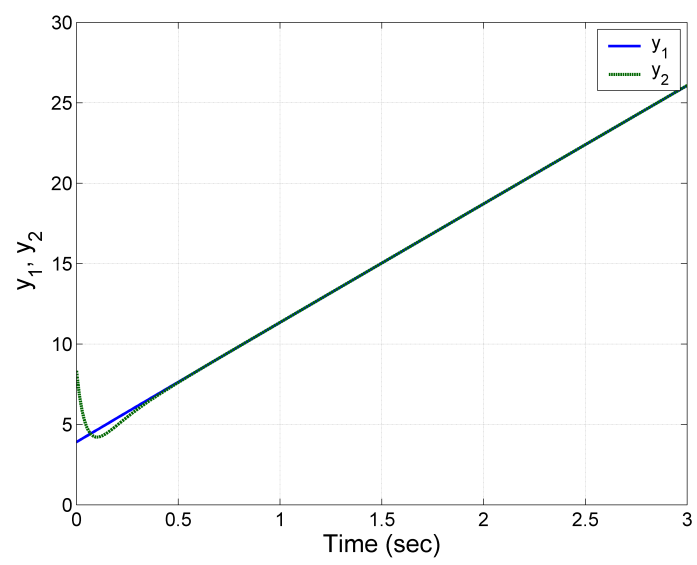

Figure 13: MATLAB simulation showing the synchronisation of the states $y_{1}$ and $y_{2}$ of the hyperjerk systems (20) and (21).

\section{Circuit realization of the new 4-D hyperjerk system with hyperchaotic attractor}

In this section, electronic circuit of the new 4-D hyperjerk system with hyperchaos attractor is designed and realized. The complete electronic circuit includes four capacitors $\left(C_{1}, C_{2}, C_{3}, C_{4}\right)$, twenty resistances $\left(R_{1}, \ldots, R_{20}\right)$, nine operational amplifiers (TL082CD), two diodes (1N4148) and three multipliers (AD633JN).

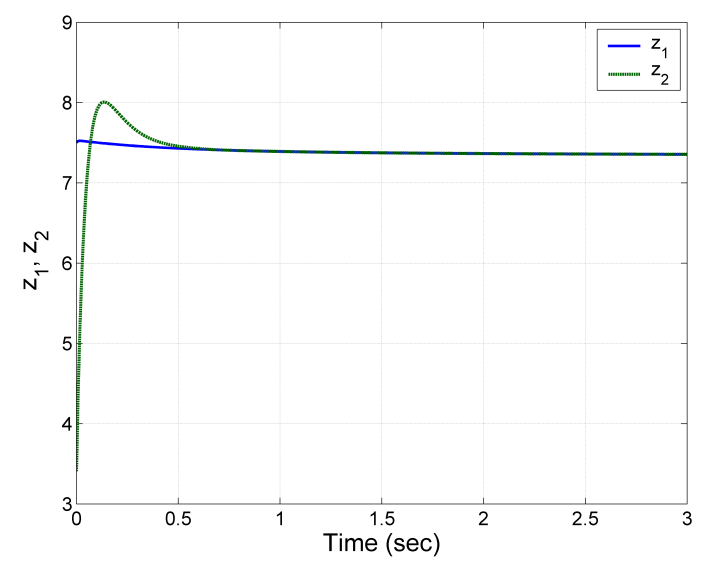

Figure 14: MATLAB simulation showing the synchronisation of the states $z_{1}$ and $z_{2}$ of the hyperjerk systems (20) and (21).

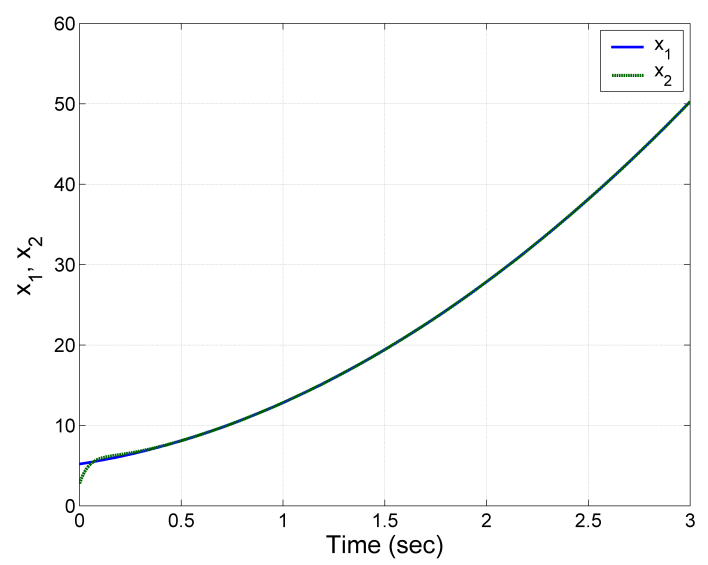

Figure 15: MATLAB simulation showing the synchronisation of the states $w_{1}$ and $w_{2}$ of the hyperjerk systems (20) and (21).

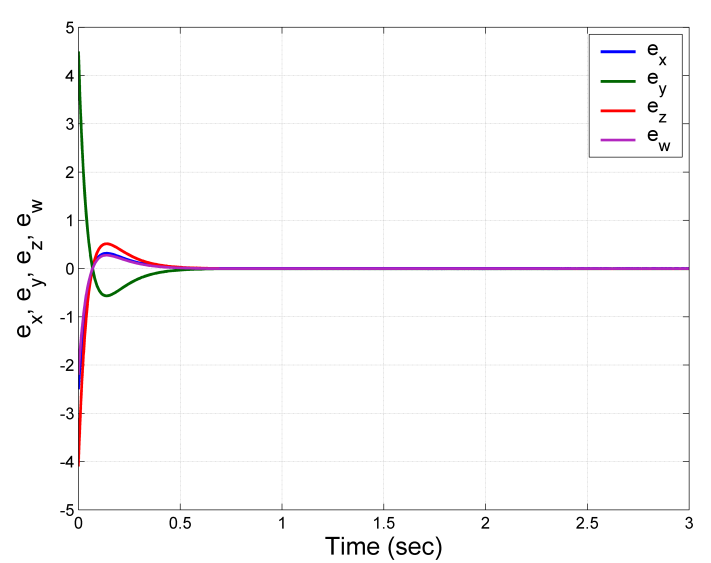

Figure 16: MATLAB simulation showing the timehistory of the synchronisation errors between the hyperjerk systems (20) and (21). 


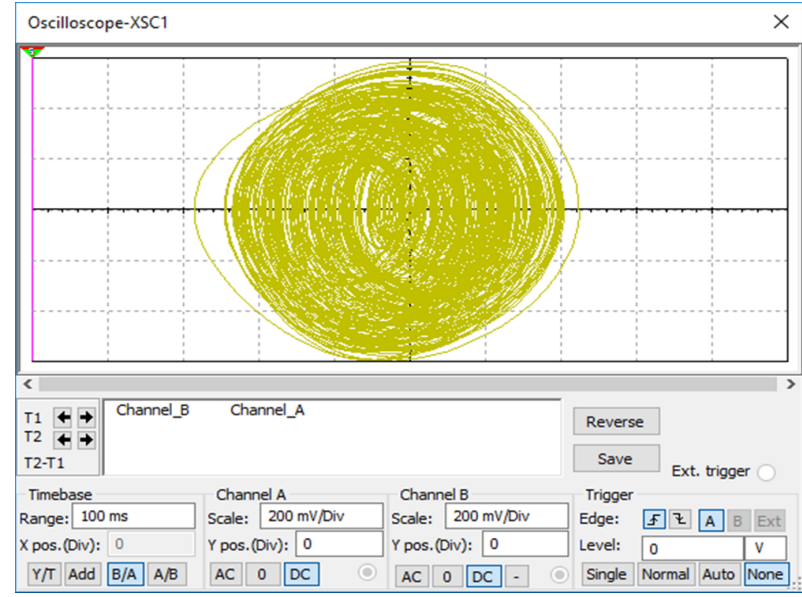

Figure 18: MultiSim circuit simulation of the hyperchaos attractor of the new 4-D hyperjerk system (30) in $x-y$ plane

By applying the Kirchhoff laws, the electronic circuit of system (4) in Figure 17 can be described by the following equations:

$$
\left\{\begin{aligned}
\dot{x}= & \frac{1}{C_{1} R_{1}} y \\
\dot{y}= & \frac{1}{C_{2} R_{2}} z \\
\dot{z}= & \frac{1}{C_{3} R_{3}} w \\
\dot{w}= & -\frac{1}{C_{4} R_{4}} x-\frac{1}{C_{4} R_{5}} y-\frac{1}{10 C_{4} R_{6}} x^{2} \\
& \quad-\frac{1}{C_{4} R_{7}} z+\frac{1}{C_{4} R_{8}} w-\frac{1}{100 C_{4} R_{9}} x^{2}|x| w
\end{aligned}\right.
$$

The signals $(x, y, z, w)$ come from Eq. (30), correspond to the state voltages of four channels, respectively. The values of all electronic components in Figure 17 are determined as: $R_{6}=33.33 \mathrm{k} \Omega, R_{7}=27.77$ $\mathrm{k} \Omega, R_{8}=1 \mathrm{M} \Omega, R_{9}=1 \mathrm{k} \Omega, R_{1}=R_{2}=R_{3}=R_{4}=$ $R_{5}=R_{10}=R_{11}=R_{12}=R_{13}=R_{14}=R_{15}=R_{16}=$ $R_{17}=R_{18}=R_{19}=R_{20}=100 \mathrm{k} \Omega$ and $C_{1}=C_{2}=C_{3}=$ $C_{4}=1 \mathrm{nF}$. The power supplies of all active devices are \pm 15 Volts. MultiSim simulation results in Figures 18-21 show hyperchaos attractor, which agree with the Matlab simulation plots shown Figures 2-5.

\section{Conclusions}

We reported a new 4-D dynamical hyperjerk system with hyperchaos with two saddle-focus rest points exhibiting Hopf bifurcation. As a control application, an integral sliding mode controller was designed for the global hyperchaos synchronisation of the new hyperjerk system with itself. Finally, a circuit model using MultiSim of the new hyperjerk system with hyperchaos was designed for practical implementation. As future work, the proposed hyperjerk system can be implemented for secure communication devices and encryption.

\section{References}

Abdelhedi, F. and Derbel, N. (2019). On the renovation and analysis of high order sliding mode approaches with application to robotic systems. International Journal of Modelling, Identification and Control, 31(4):374-381.

Ahmad, I. and Srisuchinwong, B. (2018). On the first hyperchaotic hyperjerk system with no equilibria: A simple circuit for hidden attractors. IEEE Access, 6:35449-35456.

Azar, A. T. and Zhu, Q. (2015). Advances and Applications in Sliding Mode Control Systems. Springer, Berlin, Germany.

Boulkroune, A., Hamel, S., Azar, A. T., and Vaidyanathan, S. (2016). Fuzzy control-based function synchronization of unknown chaotic systems with dead-zone input. Studies in Fuzziness and Soft Computing, 337:699-718.

Cabanas, A. M., Clerc, M. G., Laroze, D., and Leon, A. O. (2019). Chaotic patterns and localized states in spin valves. Journal of Magnetism and Magnetic Materials, 476:589-596.

Daltzis, P., Vaidyanathan, S., Pham, V.-T., Volos, C., Nistazakis, E., and Tombras, G. (2018). Hyperchaotic attractor in a novel hyperjerk system with two nonlinearities. Circuits, Systems, and Signal Processing, 37(2):613-615.

Daumann, C. C. and Rech, P. C. (2019). Hyperchaos in convection with the Cattaneo-Christov heat-flux model. European Physical Journal B, 92(5). Article ID 110 .

El-Nabulsi, R. A. (2018). Time-nonlocal kinetic equations, jerk and hyperjerk in plasmas and solar 

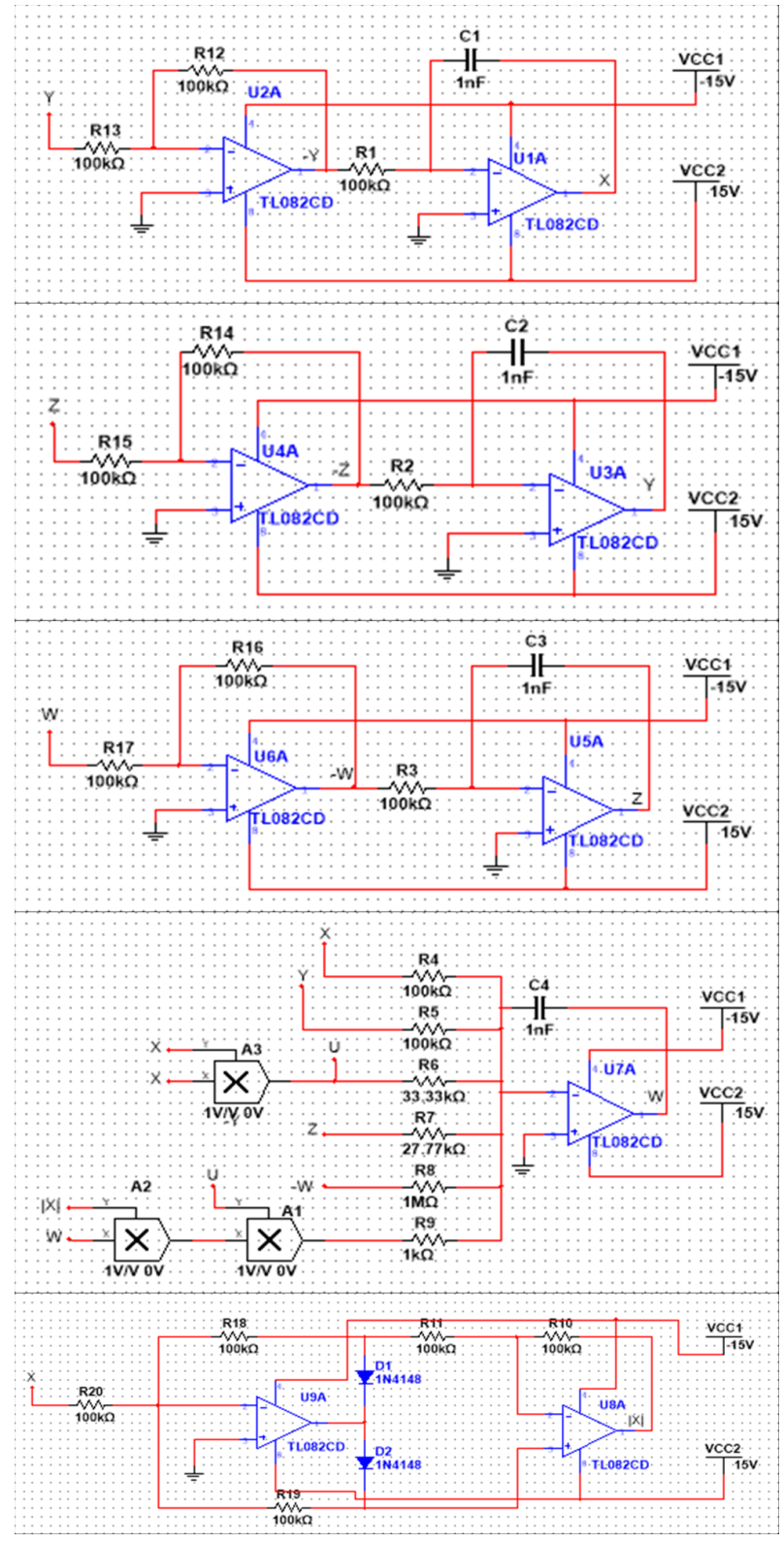

Figure 17: Circuit design of the new 4-D hyperjerk system with hyperchaotic attractor (30)

physics. Advances in Space Research, 61(12):29142931.

Fadil, H. E., Yahya, A., Oulcaid, M., Ammeh, L., and Giri, F. (2019). Output feedback nonlinear control of power system under large penetration of renewable energy sources. International Journal of Modelling, Identification and Control, 31(4):337-347.

Gatabazi, P., Mba, J. C., Pindza, E., and Labuschagne, C. (2019). Grey Lotka-Volterra models with application to cryptocurrencies adoption. Chaos, Solitons and Fractals, 122:47-57.

Ginoux, J.-M., Naeck, R., Ruhomally, Y. B., Dahuhoo, M. Z., and Perc, M. (2019). Chaos in a predatorprey-based mathematical model for illicit drug

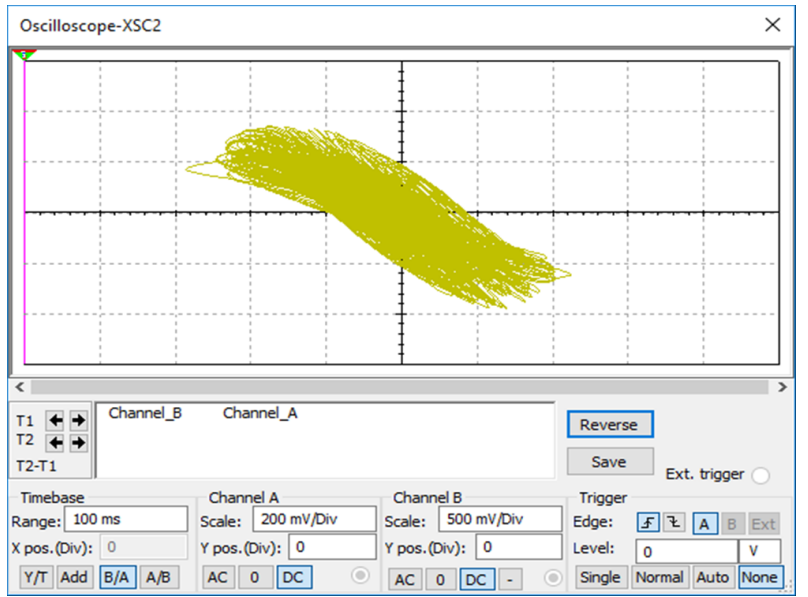

Figure 19: MultiSim circuit simulation of the hyperchaos attractor of the new 4-D hyperjerk system (30) in $x-z$ plane

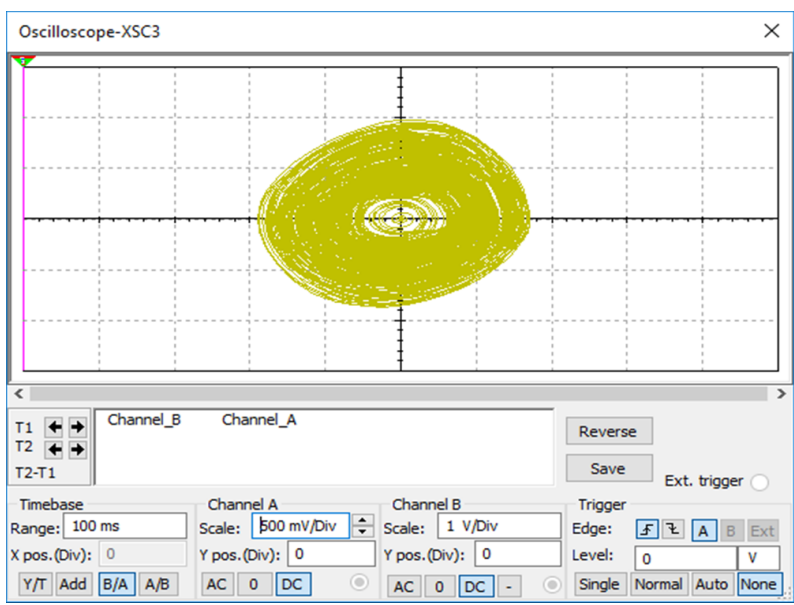

Figure 20: MultiSim circuit simulation of the hyperchaos attractor of the new 4-D hyperjerk system (30) in $z-w$ plane

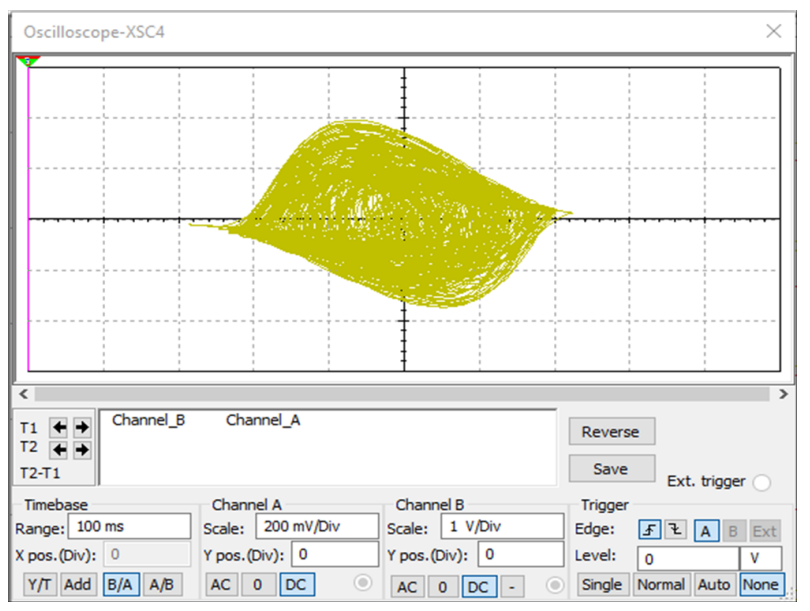

Figure 21: MultiSim circuit simulation of the hyperchaos attractor of the new 4-D hyperjerk system (30) in $x-w$ plane 
consumption. Applied Mathematics and Computation, 347:502-513.

Gritli, H. and Belghith, S. (2016). Bifurcations and chaos in the semi-passive bipedal dynamic walking model under a modified OGY-based control approach. Nonlinear Dynamics, 83(4):1955-1973.

Gusso, A., Viana, R. L., Mathias, A. C., and Caldas, I. (2019). Nonlinear dynamics and chaos in micro/nanoelectromechanical beam resonators actuated by two-sided electrodes. Chaos, Solitons and Fractals, 122:6-16.

Habrache, N., Maidi, A., and Corriou, J. (2019). Feedback control of bilinear distributed parameter system by input-output linearisation. International Journal of Modelling, Identification and Control, $31(4): 323-330$.

Jahanshahi, H., Yousefpour, A., Wei, Z., Alcaraz, R., and Bekiros, S. (2019). A financial hyperchaotic system with coexisting attractors: Dynamic investigation, entropy analysis, control and synchronization. Chaos, Solitons and Fractals, 126:66-77.

Leandro, M. A. C. and Kienitz, K. H. (2019). D-stability of parameter-dependent linear systems including discretisation by Taylor series expansion and search in a scalar parameter. International Journal of Modelling, Identification and Control, 31(4):293-302.

Lien, C.-H., Vaidyanathan, S., Yu, K.-W., and Chang, H.-C. (2019a). $\quad H_{\infty}$ performance of continuous switched time-delay systems with sector and norm bounded nonlinearities. International Journal of Modelling, Identification and Control, 31(3):229-244.

Lien, C.-H., Vaidyanathan, S., Yu, K.-W., and Chang, H.-C. (2019b). Robust mixed performance for uncertain Takagi-Sugeno fuzzy time-delay systems with linear fractional perturbations. International Journal of Modelling, Identification and Control, 31(2):193-203.

Nwachioma, C., Humberto Perez-Cruz, J. Jimenez, A., Ezuma, M., and Rivera-Blas, R. (2019). A new chaotic oscillator - Properties, analog implementation, and secure communication application. IEEE Access, 28(5). Article ID 1950087.

Prousalis, D. A., Volos, C. K., Stouboulos, I. N., and Kyprianidis, I. M. (2018). Extreme multistability in hyperjerk memristive system with hidden attractors and its adaptive synchronisation scheme. International Journal of Simulation and Process Modelling, 13(5):433-445.

Rajagopal, K., Akgul, A., Jafari, S., Karthikeyan, A., Cavusoglu, U., and Kacar, S. (2019a). An exponential jerk system: Circuit realization, fractional order and time delayed form with dynamical analysis and its engineering application. Journal of Circuits, Systems and Computers, 28(5). Article ID 1950087.

Rajagopal, K., Khalaf, A. J. M., Wei, Z., Pham, V.-T., and Alsaedi, A. (2019b). Hyperchaos and Coexisting Attractors in a Modified van der Pol-Duffing Oscillator. International Journal of Bifurcation and Chaos, 29(5). Article ID 1950067.

Rasappan, S. and Vaidyanathan, S. (2012). Global chaos synchronization of WINDMI and Coullet chaotic systems by backstepping control. Far East Journal of Mathematical Sciences, 67(2):265-287.

Ricci, D., Romero, M., and Seron, M. (2018). Lyapunovfunction-free backstepping design with application to the Lorenz system. IFAC-PapersOnLine, 51(33):223228 .

Singh, P. P. and Roy, B. K. (2019). Microscopic chaos control of chemical reactor system using nonlinear active plus proportional integral sliding mode control technique. European Physical Journal: Special Topics, 228(1):169-184.

Sundarapandian, V. (2013). Adaptive control and synchronization design for the Lu-Xiao chaotic system. Lecture Notes in Electrical Engineering, 131:319-327.

Tirandaz, H. (2019). Complete synchronisation of supply chain system using adaptive integral sliding mode control method. International Journal of Modelling, Identification and Control, 31(4):314-322.

Tsafack, N. and Kengne, J. (2018). A novel autonomous 5-D hyperjerk RC circuit with hyperbolic sine function. Scientific World Journal, 2018. Article ID 1260325 .

Utkin, V. and Shi, J. (1996). Integral sliding mode in systems operating under uncertainty conditions. In Proceedings of 35th IEEE Conference on Decision and Control, volume 4, pages 4591-4596.

Vaidyanathan, S. (2011a). Output regulation of Arneodo-Coullet chaotic system. Communications in Computer and Information Science, 133:98-107.

Vaidyanathan, S. (2011b). Output regulation of the unified chaotic system. Communications in Computer and Information Science, 198:1-9.

Vaidyanathan, S. (2012). Adaptive controller and synchronizer design for the Qi-Chen chaotic system. Lecture Notes of the Institute for Computer Sciences, Social-Informatics and Telecommunications Engineering, 85:124-133.

Vaidyanathan, S. (2013). A ten-term novel 4D hyperchaotic system with three quadratic nonlinearities and its control. International Journal of Control Theory and Applications, 6(2):97-109. 
Vaidyanathan, S. (2015a). A novel chemical chaotic reactor system and its adaptive control. International Journal of ChemTech Research, 8(7):146-158.

Vaidyanathan, S. (2015b). A novel chemical chaotic reactor system and its output regulation via integral sliding mode control. International Journal of Chem Tech Research, 8(11):669-683.

Vaidyanathan, S. (2015c). Adaptive biological control of generalized Lotka-Volterra three-species biological system. International Journal of PharmTech Research, 8(4):622-631.

Vaidyanathan, S. (2015d). Adaptive chaotic synchronization of enzymes-substrates system with ferroelectric behaviour in brain waves. International Journal of PharmTech Research, 8(5):964-973.

Vaidyanathan, S. (2015e). Analysis, control, and synchronization of a $3-\mathrm{D}$ novel jerk chaotic system with two quadratic nonlinearities. Kyungpook Mathematical Journal, 55(3):563-586.

Vaidyanathan, S. (2015f). Dynamics and control of Brusselator chemical reaction. International Journal of ChemTech Research, 8(6):740-749.

Vaidyanathan, S. (2015g). Hyperchaos, qualitative analysis, control and synchronisation of a ten-term 4-D hyperchaotic system with an exponential nonlinearity and three quadratic nonlinearities. International Journal of Modelling, Identification and Control, 23(4):380-392.

Vaidyanathan, S. (2015h). Output regulation of the forced Van der Pol chaotic oscillator via adaptive control method. International Journal of Pharm Tech Research, 8(6):106-116.

Vaidyanathan, S. (2015i). Synchronization of Tokamak systems with symmetric and magnetically confined plasma via adaptive control. International Journal of Chem Tech Research, 8(6):818-827.

Vaidyanathan, S. (2016). A novel 3-D jerk chaotic system with two quadratic nonlinearities and its adaptive backstepping control. International Journal of Control Theory and Applications, 9(1):199-219.

Vaidyanathan, S. (2017). A new 3-D jerk chaotic system with two cubic nonlinearities and its adaptive backstepping control. Archives of Control Sciences, 27(3):409-439.

Vaidyanathan, S., Abba, O. A., Betchewe, G., and Alidou, M. (2019a). A new three-dimensional chaotic system: Its adaptive control and circuit design. International Journal of Automation and Control, 13(1):101-121.
Vaidyanathan, S., Akgul, A., and Kacar, S. (2018a). A new chaotic jerk system with two quadratic nonlinearities and its applications to electronic circuit implementation and image encryption. International Journal of Computer Applications in Technology, 58(2):89-101.

Vaidyanathan, S., Akgul, A., Kacar, S., and Cavusoglu, U. (2018b). A new 4-D chaotic hyperjerk system, its synchronization, circuit design and applications in RNG, image encryption and chaosbased steganography. European Physical Journal Plus, 2018(2). Article ID 46.

Vaidyanathan, S. and Azar, A. T. (2016). TakagiSugeno fuzzy logic controller for Liu-Chen four-scroll chaotic system. International Journal of Intelligent Engineering Informatics, 4(2):135-150.

Vaidyanathan, S., Dolvis, L. G., Jacques, K., Lien, C.-H., and Sambas, A. (2019b). A new five-dimensional fourwing hyperchaotic system with hidden attractor, its electronic circuit realisation and synchronisation via integral sliding mode control. International Journal of Modelling, Identification and Control, 32(1):30-45.

Vaidyanathan, S. and Lien, C. H. (2017). Applications of Sliding Mode Control in Science and Engineering. Springer, Berlin, Germany.

Vaidyanathan, S., Sambas, A., Kacar, S., and Cavusoglu, U. (2019c). A new finance chaotic system, its electronic circuit realization, passivity based synchronization and an application to voice encryption. Nonlinear Engineering, 8(1):193-205.

Vaidyanathan, S., Sambas, A., Mamat, M., and Sanjaya WS, M. (2017). Analysis, synchronisation and circuit implementation of a novel jerk chaotic system and its application for voice encryption. International Journal of Modelling, Identification and Control, 28(2):153-166.

Vaidyanathan, S. and Volos, C. (2016). Advances and Applications in Chaotic Systems. Springer, Berlin, Germany.

Vaidyanathan, S., Volos, C., Rajagopal, K., Kyprianidis, I., and Stouboulos, I. (2015). Adaptive backstepping controller design for the anti-synchronization of identical WINDMI chaotic systems with unknown parameters and its SPICE implementation. Journal of Engineering Science and Technology Review, 8(2):7482.

Wang, P. (2019). Research on the control algorithms of human-thinking simulated control. International Journal of Modelling, Identification and Control, 31(4):348-360.

Wang, X., Vaidyanathan, S., Volos, C., Pham, V.-T., and Kapitaniak, T. (2017). Dynamics, 
circuit realization, control and synchronization of a hyperchaotic hyperjerk system with coexisting attractors. Nonlinear Dynamics, 89(3):1673-1687.

Xu, B., Wang, G., Lu, H. H.-C., Yu, S., and Yuan, F. (2019). A memristor-meminductor-based chaotic system with abundant dynamical behaviors. Nonlinear Dynamics, 96(1):765-788. 